\title{
Informe bibliográfico sobre la obra de Martin Meidegger
}

\author{
ALEJANDRO ROJAS JIMÉNEZ \\ Universidad de Málaga
}

RESUMEN

El informe presentado contiene la relación de la obra completa de Heidegger, así como una selección de monografías interesantes para su comprensión. Se ha añadido también la información sobre la edición de la correspondencia, las últimas ediciones de las obras de Heidegger traducidas al castellano y las colecciones dedicadas a la promoción de su pensamiento.

\section{PALABRAS CLAVE}

HEIDEGGER, MONOGRAFÍAS, OBRAS COMPLETAS, ESTUDIOS

\begin{abstract}
This bibliographical report contains the relation of the complete works of Heidegger, as well as a selection of interesting monographs for the understanding of his work. The report inludes the information on the edition of the correspondence, the last editions of works of Heidegger translated and the collections dedicated to the promotion of their thought.

KEYWORDS

HEIDEGGER, MONOGRAPHS, COMPLETE WORKS, STUDIES
\end{abstract}

EN ESTE INFORME BIBLIOGRÁFICO se presenta la obra completa heidegeriana según el plan de edición de Klostermann indicando los volúmenes editados, sin editar o en preparación, así como los editores. La prolífera obra de Heidegger hace de esta planificación una herramienta muy interesante para poder situar sus distintos trabajos, especialmente si tenemos en cuenta, que el orden no es cronológico, sino que fue el propio Heidegger quien ordenó y planificó esta edición pensando en sus futuros lectores. Se ha añadido a este plan de edición, también el de la correspondencia, explicitando las que han sido ya editadas y las que están en preparación.

Considerando que es fundamental disponer de una buena selcción de monografías que puedan ayudar al estudio de la obra heideggeriana, se ha añadido

\footnotetext{
C Contrastes. Revista Internacional de Filosofía, vol. XIV (2009), pp. 305-322. ISSN: 1136-4076 Licenciatura de Filosofía, Universidad de Málaga, Facultad de Filosofía y Letras Campus de Teatinos, E-29071 Málaga (España)
} 
también una interesante selección a este respecto, en la que se ha tenido cuidado de incluir aquellos trabajos que han sido dedicados al análisis de la Cuadratura, un lugar clave dentro de la obra heideggeriana y aún poco estudiado. Con esta misma intención de apuntar lugares en los que poder encontrar estudios para la comprensión de la filosofía heideggeriana, se ha creido también pertinente hacer referencia de las ediciones dedicadas a la promoción y análisis de su pensamiento: el Heidegger Studies, la Jahresgabe, la Schriftenreihe y el Heidegger-Jahrbuch.

Se informa de las últimas ediciones de las obras traducidas al castellano, y se ha creido también pertinente indicar en un último apartado los trabajos sobre la filosofía de Heidegger aparecidos en el último año siguiendo el listado que ofrece la Universidad de Friburgo, de este modo se muestra la atracción que la filosofía de Heidegger ejerce actualmente en el mundo académico internacional. Si bien, también se puede observar que en dicho interés se incide a menudo en los mismos temas quedando otros aspectos de su filosofía algo renegados a un segundo plano que realmente no es merecido, y estoy pensando concretamente en la filosofía de la Cuadratura, siendo a mi parecer la explicación directa de este desmerecimiento cierta orientación académica, que poco a poco empeiza a ser corregida, pero que durante un tiempo se olvidó del asunto del pensar propiamente filosófico reduciendo su labor al de mero juez redentor o comisario político del individuo Heidegger y su vinculación al nacionalsocialismo.

Otros repertorios bibliográficos son:

SASS, H-M. 1982: Martin Heidegger. Bibliography and Glossary, Ohio: Bowling Green University

VOLPI, F. 2005: Bibliografia, en M. Heidegger, Essere e tempo. Milán: Longanesi, pp 553-579

ESCUDERO, J.A. 2008: Apéndice bibliográfico, en M. Heidegger, El concepto de tiempo. Barcelona: Herder, pp 105-151. Herder ofrece versión en PDF on-line en:

http://www.herdereditorial.com/descargas/programa\%20filosofico\%20he idegger.pdf

BAST, R. A. Y DELFOSSE, H. 1979: Handbuch zum Textstudium von Martin Heideggers «Sein und Zeit». Bad Cannstatt/Stuttgart: Frommann/Holboog

Merece una mención especial la Universitätsbibliothek Freiburg: Martin Heidegger-Wegweiser durch die Heidegger-Literatur:

http://www.ub.uni-freiburg.de/referate/02/heidegger/heidegger_20.html

Y también son útiles las siguientes selecciones on-line:

- la selección de Pete: http://www.beyng.com/hbooks.html

- la recopilación de Alred Denker que se puede leer en: www.heidegger. org, y que también está disponible en pdf: 
http://www.freewebs.com/m3smg2/HeideggerBibliografie2003.pdf

- y en SIBF (Servicio de Información bibliográfica para la filosofía): Martin Heidegger. Bibliografía de y sobre con ocasión del aniversario de su nacimiento:

http://www.philosophybooks.info/Investigacion/Febrero2007/HeideggerBibliografia.html

\section{ObRa COMPLETA (GeSAMTAUSGABE)}

\section{I.1. ESCRITOS PUBLICADOS 1910-1976}

Band 1. Frühe Schriften (1912-16), editor: Friedrich-Wilhelm von Herrmann; XII, 454. 1978.

Band 2. Sein und Zeit (1927), editor: Friedrich Wilhelm von Herrmann; XIV, 586. 1977.

Band 3. Kant und das Problem der Metaphysik (1929), editor: Friedrich-Wilhelm von Herrmann; XVIII, 318. 1991.

Band 4. Erläuterungen zu Hölderlins Dichtung (1936-1968), editor: FriedrichWilhelm von Herrmann; 208. 1981; segunda edición en 1996; tercera edición en preparación.

Band 5. Holzwege (1935-1946), editor: Friedrich-Wilhelm von Hermann; $1^{\text {a }}$ edición 1977. $2^{\text {a }}$ edición 2003. VIII, 382.

Band 6.1 Nietzsche 1. (1936-1939), editora: Brigitte Schillbach; 1996. XIV, 596.

Band 6.2 Nietzsche 2. (1939-1946), editora: Brigitte Schillbach; 1997. VIII, 454.

Band 7. Vorträge und Aufsätze (1936-1953), editor: Friedrich-Wilhelm von Herrmann; 2000. VIII, 298.

Band 8. Was heisst Denken? (1951-1952), editora: Paola-Ludovika Coriando; 2002. VIII, 268.

Band 9. Wegmarken (1919-1958), editor: Friedrich-Wilhelm von Herrmann; $1^{\mathrm{a}}$ edición 1976; $2^{\text {a }}$ edición 1996. X, 488; $3^{\text {a }}$ edición 2004. X, 488.

Band 10. Der Satz vom Grund (1955-1956), editora: Petra Jaeger; 1997. VIII, 192.

Band 11. Identität und Differenz (1955-1957), editor: Friedrich-Wilhelm v. Herrmann; 2006. VI, 168.

Band 12. Unterwegs zur Sprache (1950-1959), editor: Friedrich-Wilhelm von Herrmann; 1985. 262.

Band 13. Aus der Erfahrung des Denkens (1910-1976), editor: Hermann Heidegger; $1^{\mathrm{a}}$ edición 1983; $2^{\text {a }}$ edición 2002. VIII, 254.

Band 14. Zur Sache des Denkens (1962-1964), editor: Friedrich-Wilhelm v. Herrmann; 2007. VI, 156

Band 15. Seminare (1951-1973): Martin Heidegger \& Eugen Fink: Heraklit - Vier Seminare - Anhang: Zürcher Seminare, editor: Curd Ochwadt; $1^{\mathrm{a}}$ edición 1986; $2^{a}$ edición 2005. 448. 
Band 16. Reden und andere Zeugnisse eines Lebensweges (1910-1976), editor: Hermann Heidegger; 2000. XXII, 842.

\section{I.2. LECCIONES 1919-1944}

\section{I.2.1. CURSOS DE MARBURGO (1923-1928)}

Band 17. Einführung in die phänomenologische Forschung (WS 1923-1924), editor: Friedrich-Wilhelm von Herrmann; $1^{a}$ edición 1994. $2^{\text {a }}$ edición 2006. XIV, 332.

Band 18. Grundbegriffe der aristotelischen philosophie (SS 1924), editor: Mark Michalski; 2002. XIV, 418.

Band 19. Platon: Sophistes (WS 1924-1925), editora: Ingeborg Schüßler; 1992. XXXII, 668.

Band 20. Prolegomena zur Geschichte des Zeitbegriffs (SS 1925), editora: Petra Jaeger; $1^{\text {a }}$ edición 1979; $2^{\text {a }}$ edición 1988; $3^{\text {a }}$ edición 1994. XII, 448.

Band 21. Logik. Die Frage nach der Wahrheit (WS 1925-1926), editor: Walter Biemel; $1^{\mathrm{a}}$ edición 1976; $2^{\mathrm{a}}$ edición 1995.VIII, 418.

Band 22. Grundbegriffe der antiken Philosophie (SS 1926), editor: Franz-Karl Blust; $1^{\mathrm{a}}$ edición 1993; $2^{\mathrm{a}}$ edición 2004. XIV, 344.

Band 23. Geschichte der Philosophie von Thomas v. Aquin bis Kant (WS 1926-1927), editor: Helmuth Vetter; 2006. XII, 256.

Band 24. Die Grundprobleme der Phänomenologie (SS 1927), editor: Friedrich-Wilhelm von Herrmann; $1^{\text {a }}$ edición 1975; $2^{a}$ edición 1989; $3^{\text {a }}$ edición 1997. X, 474.

Band 25. Phänomenologische Interpretation von Kants Kritik der reinen Vernunft (WS 1927-1928), editora: Ingtraud Görland; ${ }^{\text {a }}$ edición 1977; $2^{\text {a }}$ edición 1987; $3^{\mathrm{a}}$ edición 1995. XII, 436.

Band 26. Metaphysische Anfangsgründe der Logik im Ausgang von Leibniz (SS 1928), editor: Klaus Held; $1^{\text {a }}$ edición 1978; $2^{\text {a }}$ edición 1990; 3ª edición 2007. VI, 292.

\section{I.2.2. CURSOS DE FRIBURGO 1928-1944}

Band 27. Einleitung in die Philosophie (WS 1928-1929), editor: Otto Saame und Ina Saame-Speidel; $1^{\text {a }}$ edición 1996; $2^{\text {a }}$ edición 2001. XII, 404.

Band 28. Der deutsche Idealismus (Fichte, Hegel, Schelling) und die philosophische Problemlage der Gegenwart.(SS 1929). En anexo: Nachschrift «Einführung in das akademische Studium»(Sommersemester 1929), editor: Claudius Strube; 1997. XII, 368.

Band 29/30. Die Grundbegriffe der Metaphysik: Welt, Endlichkeit, Einsamkeit (WS 1929-1930), editor: Friedrich-Wilhelm von Herrmann; $1^{\text {a }}$ edición 1983; $2^{\mathrm{a}}$ edición 1992; $3^{\text {a }}$ edición 2004. XX, 542.

Band 31. Vom Wesen der menschlichen Freiheit. Einleitung in die Philosophie (SS -.1930), editor: Hartmut Tietjen; $1^{\mathrm{a}}$ edición 1982; $2^{\mathrm{a}}$ edición 1994. XII, 308

Band 32. Hegels Phänomenologie des Geistes (WS 1930-1931), editora: Ingtraud Görland; $1^{\text {a }}$ edición 1980; $2^{\text {a }}$ edición 1988; $3^{\text {a }}$ edición 1997. VIII, 224. 
Band 33. Aristoteles: Metaphysik IX 1-3. Von Wesen und Wirklichkeit der Kraft (SS 1931), editor: Heinrich Hüni; $1^{\text {a }}$ edición 1981; $2^{\text {a }}$ edición 1990; $3^{\text {a }}$ edición 2006. VIII, 224.

Band 34. Vom Wesen der Wahrheit. Zu Platons Höhlengleichnis und Theätet (WS 1931-1932), editor: Hermann Mörchen; $1^{a}$ edición 1988; $2^{a}$ edición 1997. X, 338.

Band 35. Der Anfang der abendländischen Philosophie (Anaximander und Parmenides) (SS 1932) (en preparacion).

Band 36/37. Sein und Wahrheit. 1. Die Grundfrage der Philosophie (SS 1933). 2. Vom Wesen der Wahrheit (WS 1933-1934), editor: Hartmut Tietjen; 2001. XIV, 306.

Band 38. Logik als die Frage nach dem Wesen der Sprache (SS 1934), editor: Günter Seubold; 1998. VIII, 176.

Band 39. Hölderlins Hymnen «Germanien» und «Der Rhein» (WS 1934-1935), editora: Susanne Ziegler; $1^{a}$ edición 1980; $2^{\text {a }}$ edición 1989; $3^{\text {a }}$ edición 1999. XII, 296.

Band 40. Einführung in die Metaphysik. (SS 1935), editora: Petra Jaeger; 1983. X, 234.

Band 41. Die Frage nach dem Ding. Zu Kants Lehre von den transzendentalen Grundsätzen (WS 1935), editora: Petra Jaeger; 1984. VIlI, 254.

Band 42. Schelling: Vom das Wesen der menschlichen Freiheit (1809) (SS 1936), editora: Ingrid Schüßler; 1988. X, 290.

Band 43. Nietzsche: Der Wille zur Macht als Kunst (WS 1936-1937), editor: Bernd Heimbüchel; 1985. XII, 298.

Band 44. Nietzsches Metaphysische Grundstellung im abendländischen Denken. Die ewige Wiederkehr des Gleichen (SS 1937), editora: Marion Heinz; 1986. VIII, 254.

Band 45. Grundfragen der Philosophie. Ausgewählte «Probleme» der «Logik» (WS 1937-1938), editor: Friedrich-Wilhelm von Herrmann; $1^{\text {a }}$ edición 1984; $2^{\text {a }}$ edición 1992. XIV, 234.

Band 46. Zur Auslegung von Nietzsches. II. Unzeitgemässe Betrachtung «Vom Nutzen und Nachteil des Historie für das Leben» (WS 1938-1939), editor: Hans-Joachim Friedrich; 2003. XII, 382.

Band 47. Nietzsches Lehre vom Willen zur Macht als Erkenntnis (SS1939), editor: Eberhard Hanser; 1989. XVI, 330.

Band 48. Nietzsche. Der Europäische Nihilismus (1940), editora: Petra Jaeger; 1986. XVI, 340.

Band 49. Die Metaphysik des deutschen Idealismus. Zur erneuten auslegung von Schelling: Philosophische untersuchungen über das Wesen der menschlichen Freiheit und die damit zusammenhängenden Gegenstände (1809), editor: Günter Seubold; $1^{\mathrm{a}}$ edición 1991; $2^{\mathrm{a}}$ edición 2006. X, 210.

Band 50. 1. Nietzsches Metaphysik(1941-1942). 2. Einleitung in die Philosopie - Denken und Dichten (WS 1944-1945), editora: Petra Jaeger; $1^{\text {a }}$ edición 1990; $2^{\text {a }}$ edición 2007. VIII, 162.

Band 51. Grundbegriffe (SS 1941), editora: Petra Jaeger; $1^{\mathrm{a}}$ edición 1981; 2a edición 1991. X, 128.

Band 52. Hölderlins Hymne «Andenken» (WS 1941-1942), editor: Curd Ochwadt; $1^{\mathrm{a}}$ edición 1982; $2^{a}$ edición 1992. X, 204. 
Band 53. Hölderlins Hymne «Der Ister» (SS 1942), editor: Walter Biemel; $1^{\text {a }}$ edición 1984; $2^{\mathrm{a}}$ edición 1993. VIII, 210.

Band 54. Parmenides (WS 1942-1943), editor: Manfred S. Frings; $1^{\mathrm{a}}$ edición 1982; $2^{\mathrm{a}}$ edición 1992. XII, 252.

Band 55. Heraklit. 1. Der Anfang des abendländischen Denkens (Heraklit) (1943) 2. Logik. Heraklits Lehre vom Logos (SS 1944), editor: Manfred S. Frings; $1^{\text {a }}$ edición 1979; $2^{a}$ edición 1987; $3^{\mathrm{a}}$ edición 1994. XII, 406.

\section{I.2.3. LECCIONES DE FRIBURGO 1919-1923}

Band 56/57. Zur Bestimmung der Philosophie. 1. Die Idee der Philosophie und das Weltanschauungsproblem (post war semester 1919); 2. Phänomenologie und transzendentale Wertphilosophie (SS 1919); 3. Anhang: Über das Wesen der Universität und des akademischen Studiums (SS 1919), editor: Bernd Heimbüchel; $1^{\text {a }}$ edición 1987; $2^{\text {a }}$ edición 1999. X, 226.

Band 58. Grundprobleme der Phänomenologie (WS 1919-1920), editor: Hans-Helmuth Gander; 1992. X, 274.

Band 59. Phänomenologie der Anschauung und des Ausdrucks. Theorie der philosophischen Begriffsbildung (SS 1920), editor: Claudius Strube; $1^{\text {a }}$ edición 1993, VIII, 202; $2^{\text {a }}$ edición 2007, VIII, 202.

Band 60. Phänomenologie des religiösen Lebens. 1. Einleitung in die Phänomenologie der Religion (WS 1920-1921). 2. Augustinus und der Neuplatonismus (SS 1921). 3. Die philosophischen Grundlagen der mittelalterlichen Mystik (WS 1918-1919), editor del 1: Matthias Jung und Thomas Regehly; editor del 2 y 3 Claudius Strube; 1995. XIV, 352.

Band 61. Phänomenologische Interpretationen zu Aristoteles: Einführung in die phänomenologische Forschung (WS 1921-1922), editores: Walter Bröcker und Käte Bröcker-Oltmanns; $1^{\mathrm{a}}$ edición 1985; 2a edición 1994 XIV, 204.

Band 62. Phänomenologische Interpretation ausgewählter Abhandlungen des Aristoteles zu Ontologie und Logik (SS 1922), editor: Günther Neumann; 2005. XXIV, 452.

Band 63. Ontologie. (Hermeneutik der Faktizität) (SS 1923). editora: Käte BröckerOltmanns; $1^{\text {a }}$ edición 1988; $2^{\text {a }}$ edición 1995. XII, 116.

\section{I.3. TRATADOS NO PUBLICADOS}

BAND 64. DER BEGRIFF DER ZEIT. (1924), editor: Friedrich-Wilhelm von Herrmann; 2004. VI, 134.

Band 65. Beiträge zur Philosophie (Vom Ereignis) (1936-1938), editor: FriedrichWilhelm von Herrmann; $1^{\text {a }}$ edición 1989; $2^{\mathrm{a}}$ edición 1994. XVI, 522; $3^{\mathrm{a}}$ edición 2003.

Band 66. Besinnung (1938-1939), editor: Friedrich-Wilhelm von Herrmann; 1997. XIV, 438. 
Band 67. Metaphysik und Nihilismus. 1. Die Überwindung der Metaphysik (1938-1939);

2. Das Wesen des Nihilismus (1946-1948), editor: Hans-Joachim Friedrich; 1999. XII, 274.

Band 68. Hegel. 1. Die Negativität. Eine Auseinandersetzung mit Hegel aus dem Ansatz in der Negativität (1938-1939); 2. Erläuterung der «Einleitung» zu Hegels «Phänomenologie des Geistes» (1942), editora: Ingrid Schüßler; 1993. X, 154; nueva edición en preparación.

Band 69. Die Geschichte des Seyns - Die Geschichte des Seyns (1938-1940) - Koinon. Aus der Geschichte des Seyns (1939), editor: Peter Trawny; 1998. XII, 230.

Band 70. Über den Anfang (1941), editora: Paola-Ludovika Coriando; 2005. XII, 200.

Band 71. Das Ereignis (1941-1942), editor: Friedrich-Wilhelm von Herrmann, en preparación.

Band 72. Die Stege des Anfangs (1944), editor: Friedrich-Wilhelm von Herrmann, en preparación.

Band 73. Zum Ereignis-Denken.

Band 74. Zum Wesen der Sprache, editor: Thomas Regehly, en preparación.

Band 75. Zu Hölderlin $\square$ Griechenlandreisen, editor: Curd Ochwadt; 2000. VI, 408.

Band 76. Zur Metaphysik - Neuzeitlichen Wissenschaft - Technik. editor: Claudius Strube, en preparación.

Band 77. Feldweg-Gespräche. (1944-1945), editora: Ingrid Schüßler; 1a edición 1995. VI, 250; $2^{\text {a }}$ edición 2007. VI, 250.

Band 78. Der Spruch des Anaximander (1946), editora: Ingeborg Schüßler, en preparación.

Band 79. Bremer und Freiburger Vorträge. 1. Einblick in das was ist. Bremer Vorträge

(1949) 2. Grundsätze des Denkens. Freiburger Vorträge (1957), editora: Petra Jaeger; $1^{\text {a }}$ edición 1994; $2^{\text {a }}$ edición 2005. VI, 182.

Band 80. Vorträge (1915-1967), editor: Hartmut Tietjen, en preparación.

Band 81. Gedachtes, editora: Paola-Ludovika Coriando; 2007. XX, 360.

\section{I.4. COMENTARIOS Y APUNTES}

BAND 82. ZU EIGENEN VERÖFFENTLICHUNGEN - ANMERKUNGEN ZU «VOM WESEN DES GRUNDES》 (1936) - Eine Auseinandersetzung mit «Sein und Zeit» (1936) - Laufende Anmerkungen zu «Sein und Zeit» (1936).

Band 83. Seminare: Platon - Aristoteles - Augustinus, editor: Mark Michalski, en preparación.

Band 84. Seminare: Leibniz - Kant, editor: Günther Neumann, en preparación.

Band 85. Vom Wesen der Sprache. Die Metaphysik der Sprache und die Wesung des

Wortes. Zu Herders Abhandlung «Über den Ursprung der Sprache», editora: Ingrid Schüßler; 1999. XII, 220.

Band 86. Seminare: Hegel - Schelling, editor: Peter Trawny, en preparación.

Band 87. Nietzsche.Seminare 1937 und 1944. 1. Nietzsches Metaphysische Grundstellung (Sein und Schein) (SS 1937); 2. Skizzzen zu Grundbegriffe des Denkens (SS 1944), editor: Peter von Ruckteschell; 2004. XXII, 324. 
Band 88. Seminare (Übungen) 1937/38 und 1941/42 1. Die metaphysischen

Grundstellungen des abendländischen Denkens 2. Einübung in das philosophische Denken, editor: Alfred Denker; 2008. XIV, 336.

Band 89. Zollikoner Seminare, editor: Claudius Strube, en preparación.

Band 90. Zu Ernst Jünger, editor: Peter Trawny; 2004. XVI, 472.

Band 91. Ergänzungen und Denksplitter.

Band 92. Ausgewählte Briefe I.

Band 93. Ausgewählte Briefe II.

Band 94. Überlegungen A.

Band 95. Überlegungen $B$.

Band 96. Überlegungen $C$.

Band 97. Anmerkungen A.

Band 98. Anmerkungen $B$.

Band 99. Vier Hefte I - Der Feldweg; Vier Hefte II - Durch Ereignis zu Ding und Welt.

Band 100. Vigiliae I, II.

Band 101. Winke I, II.

Band 102. Vorläufiges I-IV.

\section{CoRrespondencia (BRIEFWECHSEL)}

\section{II.1. EDITADAS}

HEIDEGGER-ARENDT 1998: Briefe 1925 bis 1975 und andere Zeugnisse. Edición de Ursula Ludz. Frankfurt am Main: Klostermann.

HEIDEGGER-BLOCHMANN 1989: Briefwechsel 1918-1969. Edición de Joachim W. Storck. Marbach am Neckar: Deutsche Schiller-Gesellschaft.

HEIDEGGER-BODMERSHOF 2000: Briefwechsel 1959-1976. Edición de Bruno Pieger. Stuttgart: Klett-Cotta.

HEIDEGGER-BOSS 1994: Briefe, en: Martin Heidegger, Zollikoner Seminare. Edición de Medard Boss. Frankfurt am Main: Klostermann.

HEIDEGGER-FICKER 1996: Briefwechsel 1940-1967, en: Ludwig von Ficker, Briefwechsel 1940-1967. Edición de Ignaz Zangerle, Walter Methagl y Franz Syer. Innsbruck: Haymon.

HEIDEGGER-GADAMER 1999: Heideggers Briefwechsel mit Hans-Georg Gadamer, en Jean Grondin, Hans-Georg Gadamer. Eine Biographie. Tübingen: Mohr

HEIDEGGER-HÄBERLIN 1997: Briefwechsel 1908-1960, en Paul Häberlin / Ludwig Binswanger, Briefwechsel 1908-1960. Edición de Jeannine Luczak. Basel: Schwabe.

HEIDEGGER-HUSSERL 1994: Briefwechsel 1916-1933, en: Husserliana Dokumente. Band 3. Teil 4. Die Freiburger Schüler. Edición de Karl Schuhmann y Elisabeth Schuhmann. Dordrecht: Kluwer Academic Publishers.

HEIDEGGER-JASPER 1990: Briefwechsel 1920-1963. Edición de Walter Biemel y Hans Saner. Frankfurt am Main: Klostermann. 
HEIDEGGER-KÄSTNER 1986: Briefwechsel 1953-1974. Edición de Heinrich W. Petzet. Frankfurt am Main: Insel.

HEIDEGGER-KOMMERELL 1967: Briefwechsel und Aufzeichnungen: 1914-1944, en Max Kommerell, Briefe und Aufzeichnungen: 1919-1944. Edición de von Inge Jens. Freiburg: Olten.

HEIDEGGER-LÖRITH 1984: Briefwechsel mit Heidegger, en: Karl Löwith, Zu Heideggers Seinsfrage: Die Natur des Menschen und die Welt der Natur; Sämtlichen Schriften. Bd. 8: Heidegger - Denker in dürftiger Zeit. Stuttgart: Metzler.

HEIDEGGER-RICKERT 2002: Briefe 1912-1933. Edición de Alfred Denker. Frankfurt am Main: Vittorio Klostermann.

\section{II.2. EN PREPARACIÓN}

HEIDEGGER-BULTMANN, Briefwechsel. Edición de Andreas Großmann y Klaus Müller. Frankfurt am Main: Vittorio Klostermann.

HEIDEGGER-FICKER, Briefwechsel, Edición de Bruno Pieger. Stuttgart: KlettCotta.

HEIDEGGER-JÜNGER, Briefwechsel. Edición de Günther Figal. Stuttgart: KlettCotta

HEIDEGGER-MÜLLER, Briefwechsel. Edición de Holger Zaborowski. Freiburg: Alber Verlag.

HEIDEGGER-PÖGGLER, Briefwechsel.

HEIDEGGER-WELTE, Briefwechsel. Edición de Alfred Denker y Holger Zaborowski. Stuttgart: Klett-Cotta.

\section{SELECCIÓN DE MONOGRAFÍAS SOBRE HEIDEGGER}

ASTRADA, C. 2003: Martin Heidegger: de la analítica ontológica a la dimensión dialéctica. Buenos Aires: Quadrata.

BARASH, J. A. 1995: Heidegger et son siècle. Temps de l'Etre, temps de l'histoire. Paris: Presses Universitaries de France

BARBARIC, D. (ed.) 2007: Das Spätwerk Heideggers. Ereignis - Sage - Geviert, K\&N: Würzburg.

BAST, R. A. 1986: Der Wissenschaftsbegriff Martin Heideggers im Zusammenhang seiner Philosophie. Stuttgart-Bad Canstatt: Frommann-Holzboog.

BEAUFRET, J. 1973: Dialogue avec Heidegger. I, II y III. Paris: Éditions de Minuit.

BEAUFRET, J. 1984: Entretiens avec Frédéric de Towarnicki. Paris: PUF.

BEAUFRET, J. 1986: De l'existentialisme à Heidegger. Introd. aux philosophies des l'existence et autres texts. Paris: Vrin.

BENEDITO, M. F. 1992: Heidegger en su lenguaje. Madrid: Tecnos.

BERCIANO, M. 1990: La crítica de Heidegger al pensar occidental. Salamanca: Universidad de Salamanca. 
BIEMEL, W. 1950: Le Concept de monde chez Heidegger. Louvain: Nauwelaerts.

BRIHAT, D. 1988: De l'être ou rien. Heidegger et philosophie de l'être. Paris: Tequi.

COLOMER, E. 1990: El pensamiento alemán de Kant a Heidegger, III. Barcelona: Herder.

DANNER, H. 1970: Das Göttliche und der Gott bei Heidegger. München: Anton Hain.

DE GUILEAD, R. 1965: Être et Liberté. Une étude sur le dernier Heidegger. Louvain: Nauwelaerts, Louvain.

DE WAELHENS, A. 1942: La philosophie de Martin Heidegger, Louvain: Nauwelaerts.

DE WAELHENS, A. 1953: Phénoménologie et vérité. Essai sur l'évolution de l'idée de vérité chez Husserl et Heidegger. Paris: PUF.

DIETER T. 1990: Die Zeit des Selbst und die Zeit danach. Zur Kritik der Textgeschichte Martin Heideggers. Frankfurt: Suhrkamp.

DUQUE, F. (ed) 1991: Heidegger: la voz de los tiempos sombríos. Barcelona: Serbal.

DUQUE, F. (ed.) 2008: Heidegger. Sendas que vienen, I y II. Madrid: Círculo de Bellas Artes.

DUQUE, F. 2001: En torno al humanismo. Heidegger, Gadamer, Sloterdijk. Madrid: Tecnos.

ESCUDERO, J. A. 2000: El joven Heidegger. Un estudio interpretativo de su obra temprana al hilo de la pregunta por el ser. Barcelona: Universidad Autónoma.

FIGAL, G. 1991: Martin Heidegger. Phänomenologie der Freiheit. Frankfutr am Main: Anton Hain.

FIGAL, G. 1999: Martin Heidegger zur Einführung. Hamburg: Junius.

FINK, E. 1977: Sein und Mensch. Vom Wesen der ontologischen Erfahrung. Freiburg: Alber.

GADAMER, H. G. 1983: Heideggers Wege. Studien zum Spätwerk. Tübingen: Mohr.

GARCÍA DÜTTMANN, A. 1991: Das Gedächtnis des Denkens, Versuch über Heidegger und Adorno. Frankfurt a. M.: Suhrkamp.

GETHMANN, K. F. 1974: Verstehen und Auslegung. Das Methodenproblem in der Philosophie Martin Heideggers. Bonn: Bouvier.

GETHMANN-SIEFERT, 1974: Das Verhältnis von Philosophie und Theologie im Denken Martin Heideggers. Freiburg-München: Alber.

GÖRLAND, I. 1981: Transzendenz und Selbst. Eine Phase in Heideggers Denken. Frankfurt: Klostermann.

HAAR, M. 1985: Le Chant de la terre. Heidegger et les assises de l'histoire de l'être. Paris: L'Herne.

HELTING, H. 1997: Heidegger und Maeister Eckehart. Vorbereitende Überlegungen zu ihrem Gottesdenken. Berlin: Duncker und Humbolt.

HELTING, H. 1999: Heideggers Auslegung von Hölderlins Dichtung des Heiligen. Ein Beitrag zur Grundlagenforschung der Daseinanalyse. Berlin: Duncker und Humbolt. 
JÄGER, A. 1978: Gott, Nochmals Martin Heidegger. Tübingen: Mohr.

JASPERS, K. 1989: Notizen zu Martin Heidegger. München: Piper.

KETTERING, E. 1987: Nähe. Das Denken Martin Heideggers. Pfullingen: Neske.

LACOUE-LABARTHE, 1987: La Fiction du politique. Heidegger, l'art et la politique. Paris: Bourgois.

LEIDLMAIR, K. 1991: Künstliche Intelligenz und Heidegger. Über den Zwiespalt von Natur und Geist. München: Fink.

LEYTE, A. 1995: Ensaios sobre Heidegger. Galaxia: Vigo.

LEYTE, A. 2005: Heidegger. Madrid: Alianza.

LÖTWITH, K. 1953: Heidegger, Denker ind dürftiger Zeit. Stuttgart: Fischer.

MARX, W. 1961: Heidegger und die Tradition. Eine problemgeschichtliche Einführung in die Grundbestimmungen des Seins. Stuttgart: Kohlhammer.

MATTÉI, J.F, (ed.) 2004: Heidegger. L'ènigme de l’être. Paris: PUF.

MATTÉI, J-F. 2001: Heidegger et Hölderlin. Le Quadriparti. Paris: PUF.

MERKER, B. 1988: Selbsttäuschung und Selbsterkenntnis. Zu Heideggers Transformation der Phänomenologie Husserls. Frankfurt: Suhrkamp.

PAZ RODRÍGUEZ, L. 2004: Sentido y ser en Heideger. Una aproximación al problema del lenguaje. Zaragoza: Universidad de Zaragoza.

PÖGGLER, O. 1990: Der Denweg Martin Heideggers. Pfullingen: Neske, Pfullingen.

REDONDO, P. 2005: Filosofar desde el temple de ánimo. La experiencia fundamental y la teoría del encontrarse en Heidegger. Salamanca: Universidad de Salamanca.

RICHARDSON, W. J. 2003: Heidegger. Through Phenomenology to Thought. New York: Fordham University.

RODRÍGUEZ, R. 2006: Heidegger y la crisis de la época moderna. Madrid: Síntesis.

ROJAS JIMÉNEZ, A. 2006: Hacia la Cuadratura. Málaga: Universidad de Málaga.

RORTY, R. 1991: Essays on Heidegger and others. Cambridge: Cambridge University Press.

SAFRANSKI, R. 1994: Ein Meister aus Deutschland. München: Carl Hanser.

SCHNEEBERGER, G. 1962: Nachlese zu Heidegger. Dokumente zu seinem Leben und Denken, Bern: Suhr.

SCHRÖDER, W. M., 2004: Politik des Schones. Heideggers Geviert-Konzept, politisch ausgelegt. Tübingen: Attempto.

SCHÜRMANN, R. 1982: Le Principe d'anarchie. Heidegger et la question de l'agir. Paris: Seuil.

SEGURA, C. 2007: Heidegger y metafísica. Madrid: Creative commons.

STEINER, G. 1978: Heidegger. Fontana: Brooklyn.

STUMPE, M. 2002: Geviert, Gestell, Geflecht. Die logische Struktur des Gedankens in Martin Heideggers späten Texten. Norderstedt: Braunschweig.

TOWARNICKI, F., 1999: Martin Heidegger. Souvenirs et Chroniques. Paris : Payot et Rivages.

TUGENDHAT, E., 1967: Der Wahrheitsbegriff bei Husserl und Heidegger, Berlin: Gruyter.

VATTIMO, G. 1981: Introduzione a Heidegger. Roma/Bari: Laterza. 
VATTIMO, G., 1963: Essere, storia e linguaggio in Heidegger. Torino: Edizioni di Filosofia.

VIETTA, S. 1989: Heideggers Kritick am Nationalsozialismus und an der Technik. Tübingen: Niemayer.

VITIELLO, V. 1979: Dialettica ed ermeneutica. Hegel e Heidegger. Napoli: Guida.

VOLPI, F. 1976: Heidegger e Brentano. L'aristotelesimo e il problema dell'univocità dell'essere nella formazione filosofica del Giovane Martin Heidegger. Padova: Cedam.

VOLPI, F. 1984: Heidegger e Aristotele. Padova: Daphne.

VON HERRMANN, F. W. 1981: Der Begriff der Phänomenologie bei Heidegger und Husserl. Frankfurt: Klostermann.

VON HERRMANN, F. W. 1990: Wege und Methode. Zur hermeneutischen Phänomenologie des seinsgeschichtlichen Denkens. Frankfurt: Klostermann.

VON HERRMANN, F. W. 1994: Wege ins Ereignis. Zu Heideggers Beiträge der Philosophie. Frankfurt: Klostermann.

VON HERRMANN, F. W. 1987: Hermeneutische Phänomenologie des Daseins. Eine Erläuterung von Sein und Zeit. Frankfurt: Klostermann.

WENZEL, U. 1986: Die Problematik des Gründes beim späten Heidegger. Rheinfelden: Schäuble.

ZARADER, M. 1990: Heidegger et les paroles de l'origine. Paris: Libraire philosophique.

ZIGLER, S. 1991: Heidegger, Hölderlin und die Aletheia, Martin Heideggers Geschichtsdenken in seinen Vorlesungen 1934/35 bis 1944. Berlin: Dunker und Humbolt.

ZIMMERMANN, M.E. 1990: Heidegger's Confrontation with Modernity, Bloomington: Indiana University Press.

\section{EDICIONES DEDICADAS A LA PROMOCIÓN Y ANÁLISIS DE SU PENSAMIENTO}

\section{IV.1. HEIDEGGER STUDIES}

El Heidegger Studies es una publicación anual, dedicada a la promoción de la comprensión del pensmaiento heideggeriano a través de la interpretación de sus escritos. Este anuario ofrece un foro para la interpretación fundamental de la obra de Heidegger en su conjunto cuya necesidad resulta de la publicación secuencial de las obras completas, pues los nuevos textos que aparecen en esta edición requieren, en vistas al análisis de su interés y significado, de tales estudios, que se publican en alemán, inglés y francés como corresponde al carácter internacional que poseen.

Sus números son: Heidegger Studies, 21 (2005); 20 (2004), 19 (2003); 18 (29002); 17 (2001); 16 (2000); 15 (1999); 14 (1998); 13 (1997); 12 (1996); 11 (1995); 10 (1994); 9 (1993); 8 (1992); 7 (1991); 6 (1990); 5 (1989); 3/4 (1987/1988). 


\section{IV.2. JAHRESGABEN DE LA MARTIN-HEIDEGGER-GESELLSCHAFT}

Anualmente la sociedad, con sede en Meßkirch, imprime una producción limitada y privada que envía exclusivamente a sus miembros, quedando excluidas su adquisición por personas o instituciones que no pertenezcan a la sociedad. Sus números han sido los siguientes:

Jahresgaben. 1986: Hans-Georg Gadamer: Der eine Weg Martin Heideggers.

Jahresgaben. 1987: Martin Heidegger: Das Wesen der Philosophie (Philosophie und Poesie).

Jahresgaben. 1988: Denken und Dichten bei Martin Heidegger.

Jahresgaben. 1989: Martin Heidegger: Neuzeitliche Naturwissenschaft und moderne Technik.

Jahresgaben. 1990: Verstehen und Geschehen.

Jahresgaben. 1991: Martin Heidegger: Cézanne.

Jahresgaben. 1992: Martin Heidegger: Die Armut (1943/44).

Jahresgaben. 1993: Martin Heidegger: Das Gedächtnis im Ereignis. Das Wesen des Menschen.

Jahresgaben. 1994: Martin Heidegger: Besinnung auf unser Wesen (1943/44).

Jahresgaben. 1995: Martin Heidegger: «Existenzialismus».

Jahresgaben. 1996: Hans Kock: Erinnerung an Martin Heidegger.

Jahresgaben. 1997: Martin Heidegger: Die Herkunft der Gottheit.

Jahresgaben. 1998: Martin Heidegger: Ein Brief an Ernst Jünger (18. Dezember 1950).

Jahresgaben. 1999: Hans-Georg Gadamer: Sechs Briefe an Martin Heidegger aus der Marburger Zeit.

Jahresgaben. 2000: Martin Heidegger: Rückweg und Kehre.

Jahresgaben. 2001: Martin Heidegger: Ein Brief an Friedrich Georg Jünger (19. August 1953).

Jahresgaben. 2002: Hans-Georg Gadamer: Ausgewählte Briefe an Martin Heidegger (1944-1976).

Jahresgaben. 2003: Martin Heidegger: Ausgewählte Briefe Martin Heideggers an Heinrich Wiegand Petzet (1931-1971).

Jahresgaben. 2004: Martin Heidegger: Zu E. Mörikes Gedichten «September-Morgen» und Um «Mitternacht».

Jahresgaben. 2005/06: Martin Heidegger: Ausgewählte Briefe an Hans-Georg Gadamer.

Jahresgaben. 2007: Martin Heidegger: «Kehre?» «Sagen der Kehre».

\section{IV.3. SCHRIFTENREIHE DE LA MARTIN-HEIDEGGER-GESELLSCHAFT}

Editada por Günter Figal, Hans-Helmuth Gander, Friedrich-Wilhelm von Herrmann, Manfred Riedel y Hartmut Tietjen, en Frankfurt: Klostermman.

Band I. 1992: Von Heidegger her. Wirkungen in Philosophie - Kunst - Medizin. 
Band II. 1993: Europa und die Philosophie.

Band III. 1994: «Verwechselt mich vor Allem nicht!» Heidegger und Nietzsche.

Band IV. 1997: Die Frage nach der Wahrheit.

Band V. 1998: «Herkunft aber bleibt stets Zukunft». Martin Heidegger und die Gottesfrage.

Band VI. 2000: «Voll Verdienst, doch dichterisch wohnet / Der Mensch auf dieser Erde». Heidegger und Hölderlin.

Band VII. 2005: Dimensionen des Hermeneutischen. Heidegger und Gadamer.

Band VIII. 2007: Heidegger und die Griechen.

\section{IV..4 HEIDEGGER-JAHRBUCH}

Anuario editado por Verlag Karl Alber, y con Alfred Denker y Holger Zaborowski encabezando el consejo editor.

Band I. 2004: Heidegger und die Anfänge seines Denkens.

Band II. 2005: Heidegger und Nietzsche.

Band III. 2007: Heidegger und Aristoteles.

\section{V. Últimas EDICIONES DE CADA TRADUCCIÓN DE LAS OBRAS DE HEIDEGGER AL CASTELLANO}

ACKERMANN, A. (trad.) 2003: Introducción a la metafísica. Barcelona: Gedisa. ADÁN (trad.) 2000: Correspondencia 1925-1975 y otros documentos de los legados, Barcelona: Herder.

AGOFF, I. (trad.) 2006: La pobreza. Buenos Aires / Madrid: Amorrortu.

ASPIUNZA, J. (trad.) 2006: Prologómenos para una historia del concepto de tiempo. Madrid: Alianza.

ASPIUNZA, J. (trad.) 2008: Ontología. Hermenéutica de la facticidad. Madrid: Alianza.

BARJAU, E. (trad.) 2002: Conferencias y artículos. Barcelona: Serbal.

CIRIA, A. (trad.) 2004: Lógica. La pregunta por la verdad. Madrid: Alianza.

CIRIA, A. (trad.) 2007: De la esencia de la verdad. Barcelona: Herder.

CIRIA, A. (trad.) 2007: Los conceptos fundamentales de la metafísica. Mundo-finitud - soledad. Madrid: Alianza.

DUQUE, F. (trad.) 2005: Desde la experiencia del pensar. Madrid: Abada.

DUQUE-PÉREZ (trad.) 2003: La proposición del fundamento. Barcelona: Serbal.

ESCUDERO, J. A. (trad.) 2002: Indicaciones fenomenológicas sobre Aristóteles. Madrid: Trotta.

ESCUDERO, J. A. (trad.) 2004: ¿Qué es la filosofía? Barcelona: Herder.

ESCUDERO, J. A. (trad.) 2005: La idea de la filosofía y el problema de la concepción del mundo. Barcelona: Herder.

ESCUDERO, J. A. (trad.) 2008: El concepto de tiempo. Barcelona: Herder. 
FARIAS, V. (trad.) 1991: Lógica. Lecciones de M. Heidegger. Barcelona: Anthropos. GABÁS, R. (trad.) 2005: ¿Qué significa pensar? Madrid: Trotta.

GARCÍA NORRO, J. J. (trad.) 2003: Heidegger/Jasper correspondencia 1920/63. Madrid: Síntesis.

GARCÍA NORRO, J. J. (trad.) 2008: Introducción a la investigación fenomenológica. Madrid: Síntesis.

LEYTE-CORTÉS (trad.) 1988: Identidad y diferencia. Barcelona: Anthropos.

LEYTE-CORTÉS (trad.) 1998: Caminos de bosque. Madrid: Alianza.

LEYTE-CORTÉS (trad.) 2000: Carta sobre el humanismo. Madrid: Alianza.

LEYTE-CORTÉS (trad.) 2003: ¿Qué es metafísica?. Madrid: Alianza.

LEYTE-CORTÉS (trad.) 2005: Aclaraciones a la poesía de Holderlin. Madrid: Alianza.

LEYTE-CORTÉS (trad.) 2007: Hitos. Madrid: Alianza.

MÁSMELA, C. (trad.) 2005: Parménides. Madrid: Akal.

MOLINUEVO-GARRIDO-DUQUE (trad.) 2002: Tiempo y Ser. Madrid: Tecnos.

MUÑOZ, J. (trad.) 1997: Estudios sobre mística medieval. Madrid: Siruela.

PICOTTI, D. (trad.) 2000: Hegel. Prometeo. Buenos Aires: Almagesto.

PICOTTI, D. (trad.) 2003: Aportes a la filosofía (acerca del evento). Buenos Aires: Biblos.

RAMOS, S. (trad.) 2007: Arte y poesía. Madrid: FCE.

RBIES, C. (trad.) 2003: Camino de campo. Barcelona: Herder.

REGUERA, I. (trad.) 2008: Estancias. Valencia: Pretextos.

RIVERA, J. E. (trad.) 2003: Ser y Tiempo. Madrid: Trotta.

RODRÍGUEZ, R (trad.) 1989: Autoafirmación universidad alemana. Madrid: Tecnos.

USCATESCU, J. (trad.) 2005: Introducción a la fenomenología de la religión. Madrid: Siruela.

VÁZQUEZ, M. E. (trad.) 2006: Conceptos fundamentales. Madrid: Alianza.

VÁZQUEZ-WREHDE (trad.) 2006: La fenomenología del espíritu de Hegel, Madrid: Alianza.

VERNAL, J. L. (trad.) 2005: Nietzsche. Barcelona: Destino.

XOLOCOTZI-GUTIÉRREZ (trad.) 20007: Cartas a Max Müller y Bernhard Welte. México: Universidad Iberoamericana.

ZABALETA, P. (trad.) 2005: Observaciones relativas al arte, la plástica, el espacio. Pmaplona: Universidad pública.

ZIMMERMAN, I. (trad.) 2002: De camino al habla. Barcelona: Serbal.

ZIMMERMAN, I. (trad.) 2002: Serenidad. Barcelona: Serbal.

VI. OBRAS SOBRE HEIDEGGER APARECIDAS EN EL ÚLTIMO AÑo (2008) SEGÚN EL LISTADO DE LA UNIVERSIDAD DE FRIBURGO

ACEVEDO, JORGE: Acerca de verdad y valor en Heidegger, en Observaciones Filosóficas, 6 (2008) [revista electrónica]. 
BARON, FRANK 2008: Kristeller's farewell to Heidegger and Gentile: The exile of Renaissance studies to the United States, en WULF KOEPKE-JÖRG THUNECKE (eds.), Preserving the memory of exile: Festschrift for John M. Spalek on the occassion of his 80th birthday. Nottingham: Refugium.

BIEMEL, WALTER: «Mein Lebensweg im Rückblick», en Heidegger studies Heidegger Studien - Études Heideggeriennes 24 (2008) 219-247.

BORMAN, ALEXANDRA 2008: «Die Unheimlichkeit des Daseins»: Sprache und Tod im Werk Thomas Bernhards. Eine Untersuchung anhand der Daseinsanalyse Martin Heideggers. Hamburg: Kovač, 2008.

COLONY, TRACY: «The wholly other: Being and the last God in Heidegger's Contributions to philosophy», en Journal of the British Society for Phenomenology, 39 (2008) 186-199.

DALISSIER, MICHEL: «La notion de "comportement" selon Heidegger», en Revue philosophique de Louvain, 106 (2008) 270-303.

DONOHOE, JANET: «The place of tradition: Heidegger and Benjamin on technology and art», en Journal of the British society for phenomenology 39 (2008), S. 260274.

FÉDIER, FRANÇOIS: «En Rusia», trad. Acevedo, Jorge, Sologuren, Jaime en Revista de Filosofía 64 (2008) 231-247. Traducción de: Fédier, François: «En Russie», en Entendre Heidegger et autres exercices d'écoute, Éditions Le Grand Souffle, Paris, 2008. S. 331-358.

FÉDIER, FRANÇOIS 2008: «En Russie», en FÉDIER, FRANÇOIS 2008: Entendre Heidegger et autres exercices d'écoute. Paris: Éditions Le Grand Souffle, Paris.

FEHÉR, ISTVÁN: «Religion, Theologie und Philosophie auf Heideggers Weg zu Sein udn Zeit: Das Phänomenologische, das Hermeneutische, das Faktische und das Historische mit Blick auf Dilthey und das Urchristentum», en Heidegger studies - Heidegger Studien - Études Heideggeriennes, 24 (2008) 103-144.

GERL-FALKOVITZ, HANNA-BARBARA: «Romano Guardini und Martin Heidegger: Person als Antwort auf Existenz?», en CHIRSTIAN SCHALLER (ed) 2008: Mittler und Befreier: Die christologische Dimension der Theologie. Freiburg i. Br: Herder.

GLOY, KAREN: «Heideggers Zeittheorie», en KAREN GLOY (ed) 2008: Philosophiegeschichte der Zeit. München: Fink.

GRIESSER, WILFRIED: «Zeit und Zeitlichkeit beim späten Paul Natorp und bei Heidegger», en Philosophisches Jahrbuch, 115 (2008) 261-287.

GROSSMANN, ANDREAS: «Rhetorik und Politik: Zu einer unausgetragenen Kontroverse zwischen Hannah Arendt und Martin Heidegger», en Philosophsiches Jahrbuch 115 (2008) 314-327.

HERRMANN, FRIEDRICH-WILHELM VON: «Topologie und Topographie des Nihilismus aus dem Gespräch zwischen Ernst Jünger und Martin Heidegger», en Heidegger studies - Heidegger Studien - Études Heideggeriennes 24 (2008) 21-37.

HINLICKY, PAUL R.: «Luther and Heidegger», en Lutheran quarterly 22 (2008) 78-86. 
HÖRL, ERICH: «Die offene Maschine. Heidegger, Günther und Simondon über die technologische Bedingung», en Modern Language Notes, 123, 3 (2008) 632-655.

HÜBNER, HANS: «Grundstrukturen von Heideggers Denken: Zu Cathrin Nielsens "Die entzogene Mitte - Gegenwart bei Heidegger"», en Heidegger studies Heidegger Studien - Études Heideggeriennes, 24 (2008) 207-214.

HUAIYU, WANG: «Care and Reverence: Exploring the Origin of Early Confucian Thinking», en Journal of Chinese Philosophy, 35 (2008) 139-157.

KALARY, THOMAS: «New access to Being and time: Focusing on Friedrich-Wilhelm von Herrmann's commentary on Sein und Zeit», en Heidegger studies - Heidegger Studien - Études Heideggeriennes, 24 (2008) 183-206.

KIENZLER, WOLFGANG: «Kuinzig - Heideggers Umgang mit einem Wort», en Allgemeine Zeitschrift für Philosophie, 33 (2008) 191-194.

KOVACS, GEORGE: «Heidegger's directives in mindfulness for understanding the be-ing-historical relationship of machination and art», en Heidegger studies Heidegger Studien - Études Heideggeriennes, 24 (2008) 39-59.

MA, LIN: «The mysterious relations to the east», en Journal of the British society for phenomenology, 39 (2008) 275-292.

MAFFESOLI, MICHEL: «La connivence impensée: Martin Heidegger et Claude Lévi-Strauss», en La magazin littéraire, 475 (2008) 80-81.

MARION, JEAN-LUC: «Remarques sur les origines de la Gegebenheit dans la pensée de Heidegger», en Heidegger studies - Heidegger Studien - Études Heideggeriennes, 24 (2008) 167-179.

MACGRATH, SEAN J.: «Alternative confessions, conflicting faiths: a review of «The influence of Augustine on Heidegger»», en American catholic philosophical quarterly, 82 (2008) 317-335.

MCGUIRK, JAMES N.: «Aletheia and Heidegger's transitional readings of Plato's cave allegroy», en Journal of the British Society for Phenomenologie, 39 (2008) $167-185$.

MARTINO, ANTONIO: «Rivisitazioni heideggeriane: senso e struttura della cura: il concetto di temporalità che scaturisce dalla "Vorlaufende Entschlossenheit"», en Antonianum, 83 (2008) 41-51.

MEI, TODD S.: «Heidegger and Teilhard de Chardin: the convergence of history and future», en Modern theology, 24 (2008) 75-101.

O'MURCHADHA, FELIX: «Reduction, Externalism and Immanence in Husserl and Heidegger», en Synthese: An International Journal for Epistemology, Methodology and Philosophy of Science, 160 (2008) 375-395.

PIPER, ARTHUR: «Beauty and Truth in Science and Phenomenology», en Analecta Husserliana: The Yearbook of Phenomenological Research, 97 (2008) 225238.

PÖGGELER, OTTO: «Die Mittagslinie Paul Celan und Martin Heidegger», en EDITH DÜSING (ed) 2008: Geist und Literatur: Modelle in der Weltliteratur von Shakespeare bis Celan. Würzburg: Königshausen \& Neumann.

RADLOFF, BERNHARD: «Machination and the political in Heidegger's mindfulness», en Heidegger studies - Heidegger Studien - Études Heideggeriennes, 24 (2008) 145-166. 
REHBERG, ANDREA: «Finding a place for spache», en Journal of the British Society for Phenomenology, 39 (2008) 103-107.

RENTSCH, THOMAS: «Martin Heidegger (1889-1976)», en OTFRIED HÖFFE (ed) 2008: Klassiker der Philosophie, Bd. 2: Von Immanuel Kant bis John Rawls. München: C. H. Beck.

RICCI, FELICETTI: «Gagner la Heimatlosigkeit», en Heidegger studies - Heidegger Studien - Études Heideggeriennes, 24 (2008) 61-102.

ROESNER, MARTINA: «Das große Spiel der Epoché: Die transzendentalphänomenologische Einstellung zwischen natürlichem Weltverhalten und theoretischer Wissenschaft», en Husserl studies, 24 (2008) 31-52.

RUHSTORFER, KARLHEINZ: «Die Erwartung des Sterblichen - Martin Heidegger», en KARLHEINZ RUHSTORFER 2008: Christologie. Paderborn: Schöningh.

RUSSELL, MATHESON: «Phenomenological reduction in Heidegger's Sein und Zeit: A new proposal», en Journal of the British society for phenomenology, 39 (2008) 228-248.

SCHERB, JÜRGEN L.: «Nichtet das Nichts wirklich nicht?: Analyse und Explikation; oder: eine deutsche Vorkriegsdebatte europäisch belichtet», en Philosophisches Jahrbuch, 115 (2008) 77-98.

SCHÜRCH, FRANZ-EMMANUEL: «Le sens de la négativité dans l'à $\lambda \hat{\theta} \theta \varepsilon \iota \alpha$ : Heidegger et les deux voiles», en Revue philosophique de Louvain, 106 (2008) 304-329.

STAEHLER, TANJA: «Unambiguous calling? Authenticity and ethics in Heidegger's Being and time», en Journal of the British society for phenomenology, 39 (2008) 293-313.

THOMÄ, DIETER: Ausflug in den Abgrund. In: Literaturen Nr. 4 (2008), S. 26-28 . - Anläßlich von E. Faye: Heidegger: Die Einführung des Nationalsozialismus in die Philosophie. 2008.

TOUSSAINT, STÉPHANE: «L'origine heideggerienne et l'inhumain» en STÉPHANE TOUSSAINT 2008: Humanismes - antihumanismes: De Ficin à Heidegger. Paris: Les belles lettres.

WELZ, CLAUDIA: «God - a phenomenon?: Theology as semiotic phenomenology of the invisible», en Studia theological, 62 (2008) 4-24.

XOLOCOTZI, ÁNGEL: «La síntesis fenómenológica» en Gregorianum, 89 (2008) 332-346.

ZIAREK, KRZYSZTOF: «The return to philosophy? or: Heidegger and the task of thinking», en Journal of the British society for phenomenology, 39 (2008) 249259. 